\title{
PAPER
}

\section{Use of neuronavigation and electrophysiology in surgery of subcortically located lesions in the sensorimotor strip}

\author{
W Eisner, J Burtscher, R Bale, R Sweeney, F Koppelstätter, S Golaszewski, C Kolbitsch, \\ K Twerdy
}

See end of article for

authors' affiliations

Correspondence to Dr Wilhelm Eisner,

Neurosurgical Department, University of Innsbruck,

Anichstrasse 35, A-6090

Innsbruck, Austria;

wilhelm.eisner@uklibk.ac.at

Received 12 march 2001

In revised form 13

Novenber 2001

Accepted

13 November 2001
J Neurol Neurosurg Psychiatry 2002;72:378-381

Objectives: Subcortical lesions in the sensorimotor strip are often considered to be inoperable. The purpose of this study was to evaluate the usefulness of a combined approach for surgery in this region, aided by a robotic neuronavigation system under electrophysiological control.

Methods: In a prospective study on 10 patients, space occupying lesions in the sensorimotor central area were removed using the Surgiscope ${ }^{\circledR}$ robotic navigation system and the Nicolet Viking IV $®$ electrophysiological system.

Results: Precise fumour localisation with the neuronavigation system and the information on the patient's cortical motor distribution obtained by bipolar cortical stimulation led to postoperative improvement in motor function in all but one patient. Seven of the patients had focal, defined pathology (four metastases; two cavernoma; one aspergilloma).

Conclusion: Due to the implementation of two recent technologies, surgery of lesions in the subcortical sensorimotor region can be performed with greater confidence.
$\mathrm{T}$ he removal of space occupying lesions in the sensory motor region carries a considerable risk of postoperative paresis. ${ }^{1}$ This is partly because intraoperative motor function mapping has shown great variability in the representation of motor function on the cortical surface. ${ }^{23}$ This study evaluates the usefulness of combining robotic neuronavigation ${ }^{4}$ to approach subcortically located lesions. Robotic neuronavigation compensates for the loss of anatomical landmarks ${ }^{4}$ as does stereotactically guided surgery. ${ }^{5}$ Direct, bipolar cortical electrophysiological mapping informs us about the motor functionality of the exposed subcortical structures.

\section{PATIENTS AND METHODS}

From April 1999 until July 2000, a total of 32 patients with space occupying lesions in or around the sensorimotor region were operated on. Twenty two of these lesions could be localised without neuronavigation due to their location in the outer cortex. The remaining 10 patients (men/women=9/1; age 25 to 66 years, mean: 43 years) are the topic of this study. The depths of tumour localisation was 8 to $20 \mathrm{~mm}$ in four patients and between 25 and $55 \mathrm{~mm}$ in six patients. The diameter of the lesions was less than $10 \mathrm{~mm}$ in four patients, between 10 and

Table 1 Localisation of the lesions according to the stimulation results

\begin{tabular}{|c|c|c|}
\hline Motor response & $\begin{array}{l}\text { Number of } \\
\text { patients and } \\
\text { function in } \\
\text { cortex }\end{array}$ & $\begin{array}{l}\text { Number of patients and } \\
\text { function in resection cavity }\end{array}$ \\
\hline $\begin{array}{l}\text { Contralateral upper } \\
\operatorname{limb}\end{array}$ & 6 & 6 \\
\hline $\begin{array}{l}\text { Contralateral lower } \\
\operatorname{limb}\end{array}$ & 0 & 2 \\
\hline $\begin{array}{l}\text { Combined upper and } \\
\text { lower limb }\end{array}$ & 1 & 1 \\
\hline Face & 0 & $\begin{array}{l}\text { One patient had no motor } \\
\text { function in the resection cavity }\end{array}$ \\
\hline Face and upper limb & 3 & 0 \\
\hline
\end{tabular}

$20 \mathrm{~mm}$ in four patients, and two patients had $20-30 \mathrm{~mm}$ lesions. According to neuroradiological diagnostics, lesions were located in the precentral gyrus in five patients, in the postcentral gyrus in three patients, and beneath the central sulcus in two patients. No lesion was located in the superior frontal gyrus or in the supplementary motor area. Six lesions were located in the right hemisphere. All 10 patients were right handed and, according to the Oldfield's handedness test, ${ }^{7}$ left hemisphere dominant. No patient presented with primary or postictal aphasic disturbance.

\section{Procedure}

On the day of or the day before surgery, contrast enhanced sagittal 3D-MPRage MRI was performed (TR/TE/ $=9.7 \mathrm{~ms} / 4$ $\mathrm{ms} / 12^{\circ}$, matrix $=256 \times 256$, voxel dimension $1.4 \times 1 \times 1 \mathrm{~mm}^{3}$, $\mathrm{FoV}=250 \mathrm{~mm}$, effective thickness $=1.4 \mathrm{~mm}$, slice thickness $=165 \mathrm{~mm}$, number of slabs $=1$, number of partitions $=114$ ). Eight skin markers were glued on the patient's head before MRI (preferably in locations with little skin displacement) and remained there until surgery. In case a skin marker was lost or displaced, the circumference of each marker was marked with a water resistant pen. The preoperative MRI data set was entered in the Surgiscope ${ }^{\circledR}$ neuronavigation system (Fa JoJuMarie, Berlin, Germany) located in the operating theatre. ${ }^{4-10}$ As in stereotactic procedures, the access route to the target point was planned in various trajectories through the cortex preoperatively in the absence of the patients.

After induction of anaesthesia ( $3 \mathrm{mg} / \mathrm{kg}$ propofol and $3 \mu \mathrm{g} /$ $\mathrm{kg}$ fentanyl) and orotracheal intubation $(0.6 \mathrm{mg} / \mathrm{kg}$ rocuronium), propofol infusion $(4-6 \mathrm{mg} / \mathrm{kg} /$ hour $)$ was used for maintainance of total intravenous anaesthesia. After registration of the patient in the operation room, using the bi-directional robotic microscopic system SurgiScope ${ }^{\circledR}$ (Fa JoJuMarie, Berlin, Germany) the trajectory to the target point

Abbreviations: TIVA, total intravenous anaesthesia; fMRI, functiona magnetic resonance imaging 
was localised by a non-sterile probe and the site of the craniotomy was drawn on the skin of the patient. Under sterile conditions, registration was controlled by a sterile probe to identify shifts of the head caused by manipulation at the beginning of surgery or by the draping process with respect to the dynamic reference frame mounted on the Mayfield head clamp. Then the craniotomy was performed and the cortex was exposed. The Surgiscope indicated the site of the tumour by laser guidance. ${ }^{11}$

Train of Four peripheral nerve stimulation producing muscle contraction equal to or higher than $90 \%$ was required before motor-cortical stimulation (mapping) was started. All patients were operated on with the aid of motor cortex mapping using Penfield's ${ }^{12}$ procedure of direct cortical stimulation, modified by one of us (WE), ${ }^{3}$ using general, anaesthesia without neuromuscular blocking agents. For intraoperative identification of the sensorimotor region, direct electrical cortical stimulation $^{2}$ of the exposed structures is best. It is the oldest and for our purpose most effective technique. Direct stimulation gives information on the functional distribution of motor cortex function and descending fibre tracts. ${ }^{313}{ }^{14}$ Electrophysiological mapping was carried out using a Nicolet Viking IV device. A Codman bipolar forceps or an Inomed bipolar stimulation probe (Fa Inomed, Tenningen, Germany) was used. Direct cortical bipolar stimulation was performed using a square wave impulse with $0.2 \mathrm{~ms}$ duration and 50 stimuli/ second, incrementally increasing intensities from 5 to $40 \mathrm{~mA}$. Bipolar stimulation defines an activated area between the tips of the stimulation probes. By contrast with monopolar stimulation, the stimulation area is better defined between the tips of a forceps than by the circular stimulated area surrounding the tip of a monopolar electrode. The stimulation points were marked by sterile Roman numbers. Subsequently, each point was stimulated with the same intensity starting with $5 \mathrm{~mA}$, while observing the evoked phenomena in the limbs and face of the patients. The laser guide of the Surgiscope allows comparison of tumour location and the distribution of cortical areas with motor function. Both the results of tumour location and of functional mapping were taken into consideration to create a so-called "silent" approach or a controlled dissection of motor fibres to approach the lesion. Through a minicorticotomy the subcortically located lesion was removed while continuously directly stimulating the transcortical approach, identifying the descending pyramidal fibre tracts. In every patient histopathological examination of the tumour and the surrounding structures was performed to ensure a radical resection.

\section{RESULTS}

The histopathological evaluation of the resected tumours showed metastases (four patients), cavernous angiomas (two patients), and one patient each with aspergillus granuloma, low grade oligoastrocytoma World Health Organisation (WHO) II, astrocytoma WHO grade III, and a cystic lesion as a demyelinisation focus. A gross total tumour resection could be achieved in eight patients. A small ( $3 \mathrm{~mm}$ diameter) tumour particle remained between the descending pyramidal fibres in the patient with the astrocytoma WHO grade III. Radiological control examinations every 3 months show no tumour progression to date.

All patients were clinically evaluated preoperatively and postoperatively, as well as 1 and 4 weeks after surgery. Preoperative clinical motor grading showed normal findings (MRC grade 5/5) in five patients with unchanged postoperative examination. Five patients showed slight to medium severe motor deficits preoperatively (MRC grade $4 / 5$ or $3 / 5$ ). All but one patient had a motor benefit after surgery. In three patients a motor improvement was noted l week after surgery. One patient improved to normal motor function in the follow up examination 4 weeks after surgery. One patient showed a

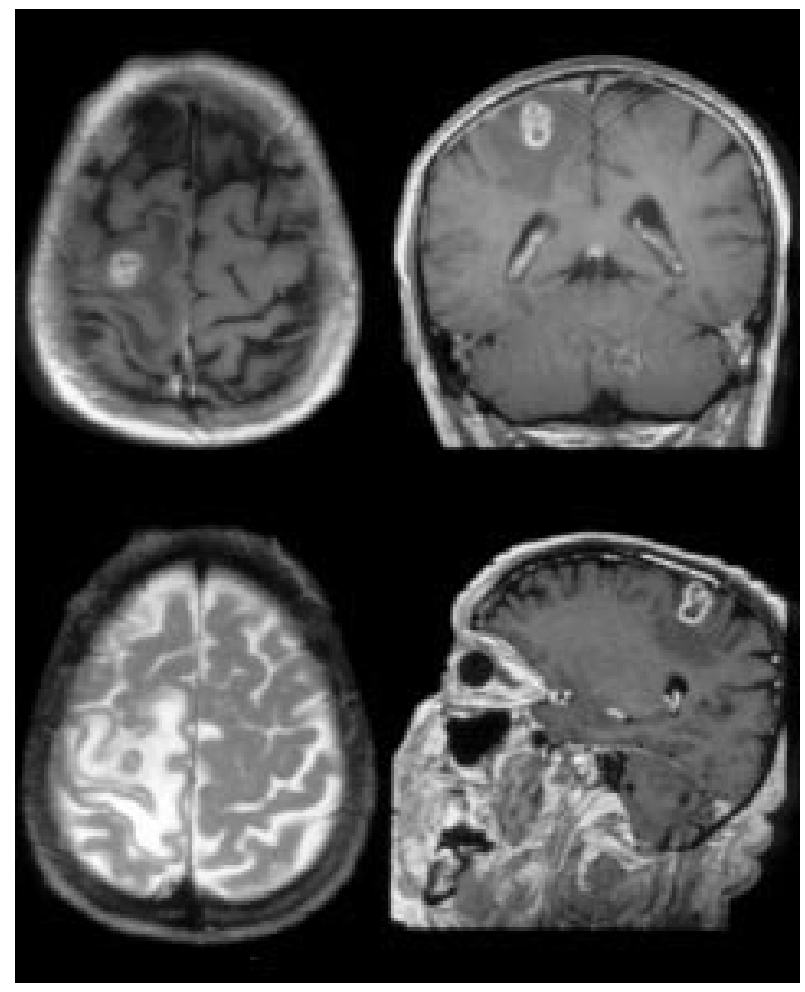

Figure 1 Preoperative MRI, axial, coronar coronal and sagittal T1 weighted images (TR/TE $\left./ \square=800 \mathrm{~ms} / 12 \mathrm{~ms} / 90^{\circ}\right)$ with contrast amplification and axial T2 weighted image (TR/TE/a $=2000$ $\left.\mathrm{ms} / 90 \mathrm{~ms} / 90^{\circ}\right)$.

preoperative motor deficit in hip function. After surgery the hip bending function was normalised but a new foot elevation paresis appeared. With the aid of an orthopaedic device to stabilise the right foot the patient was again able to walk. This patient therefore had permanent neurological deterioration after radical tumour resection, verified intraoperatively by cytological examination and postoperatively by CT and contrast enhanced T2 and T1 weighted MRI.

One patient had a subcutaneous suture granuloma. No intraoperative or postoperative bleeding or infections appeared in these patients.

A total of 73 positive stimulation sites were elicited in these 10 patients; of these, 55 sites were located in the precentral gyrus and 18 in the postcentral gyrus.

The results of the cortical stimulation are shown In table 1. In three patients the stimulation of the tumour resection cavity differed from those of the cortical stimulation site. Two patients had their cortical stimulation areas in the face and upper limb, whereas their resection cavity showed function of the lower limb. One patient did not exhibit a cortical hand area despite a large cortical exposure. The cortical stimulation in that patient gave us many stimulation points with motor function of the face, tongue, and jaw, adjacent to a functional gap without any cortical motor function. On the other side of this gap we could then stimulate the proximal upper limb and the shoulder. According to Penfield's homunculus ${ }^{9}$ the motor hand area should have been where the silent motor area was. This motor hand area was not displaced to the frontal gyri gyrus or to the parietal cortex. The lesion was approached through a non-motor cortical area disclosing a cyst filled with trabecels or fibres. A biopsy was taken after stimulating the wall of the cavity and, surprisingly, finding all motor function of the hand, which was missing on the cortex.

Three patients had a disturbance of sensory function preoperatively. Two of these patients had tingling paraesthesias in the contralateral arm, hand, and face preoperatively, 


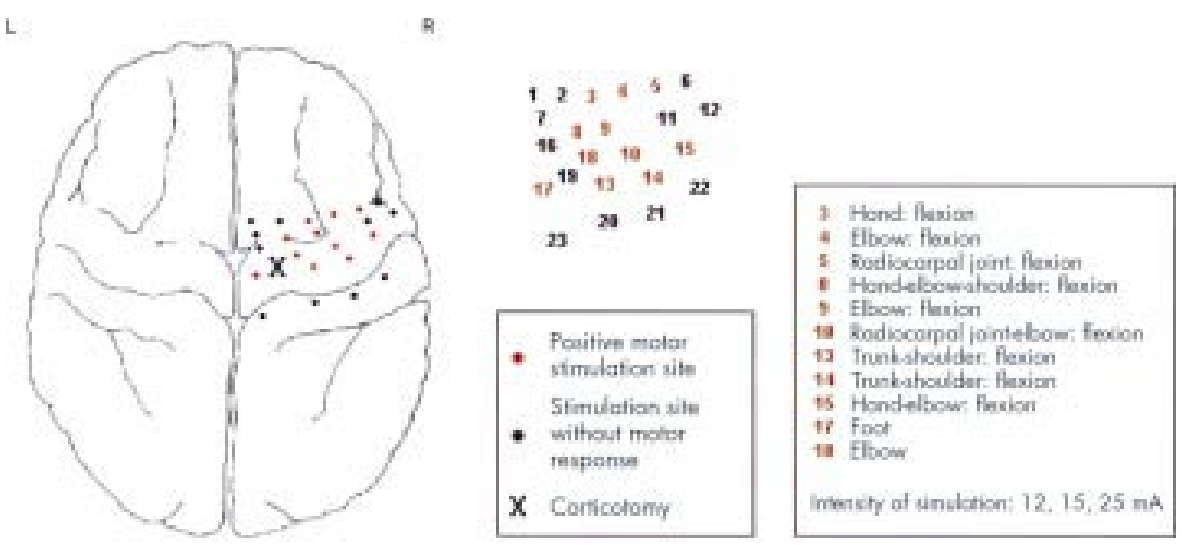

Figure 2 Patient's intraoperative situation and area of corticotomy $(x)$.

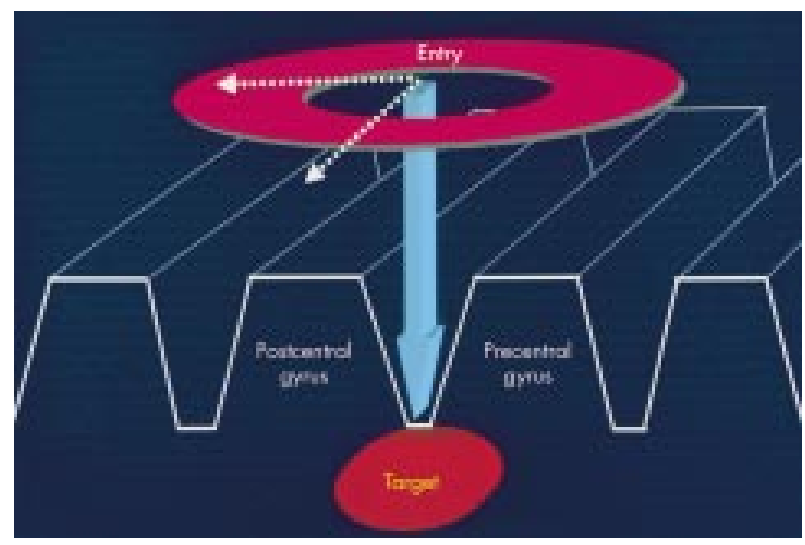

Figure 3 Spherical mode of the Surgiscope working as an invisible centre of arc stereotactic system.

which completely resolved postoperatively. In their follow up examinations, no sensory deficit could be found. One patient had a cavernous malformation in the postcentral gyrus. Surgery was performed via the intraparietal sulcus. The second patient had an oligoastrocytoma WHO II and the patient was operated on through the central sulcus. Before surgery the patient additionally had a paresis, which completely resolved after surgery. The third patient with a demyelinating focus had a slight paresis in the left hand preoperatively and hypaesthesia in the left hand. Both symptoms disappeared after surgery.

\section{CASE REPORT}

A 60 year old patient was admitted to the neurological department after his first epileptic seizure under immunosuppressive medication 1 year after liver transplantation because of a primary liver carcinoma. Neuroradiological evaluation disclosed a central necrotic/hypointense lesion with surrounding contrast enhancement (fig l). A metastasis, or abscess, or primary brain tumour were included in the differential diagnosis. Blood and CSF examination were normal. After craniotomy the cortical surface showed motor function everywhere with the exception of a $5 \mathrm{~mm}$ square (fig 2). By contrast with what was to be expected from the the Tl contrast amplified enhanced MRI, the space occupying lesion could not be seen on the cortical surface. Using the spherical mode (centred movement around a target) of the Surgiscope, targeting was modified (fig 3). The tumour was removed radically without "touching" its capsula. The neural tissue surrounding the tumour consisted of pyramidal fibres with motor function of the contralateral lower limb at the medial wall and motor function of the contralateral shoulder at the lateral wall. Postoperativly, the patient had a transient weakness in shoulder elevation of the left arm. That deficit was due to manipulation, not due to destruction of pyramidal fibres because the patient was free of neurological deficits 1 week after surgery. Histopathological examination showed aspergilloma. The patient is currently alive more than 24 months after surgery.

\section{DISCUSSION}

We have shown that electrophysiologically controlled surgery by bipolar electric stimulation of the cerebral cortex and descending fibre system is a very effective instrument for detecting cerebral functions in exact spatial distribution. For intraoperative identification of the sensorimotor region, either direct electrical cortical stimulation ${ }^{2}$ of the exposed structures or the stimulation of a peripheral nerve (median nerve or tibial nerve), recording the indirectly evoked potentials from the cortex, ${ }^{15}$ can be used. The value of motor cortex mapping is higher than by cortical somatosensory evoked potentials and phase reversal phenomena in the rolandic sulcus after peripheral nerve stimulation. ${ }^{16}$ Additionally, phase reversal recording offers no information on the particular distribution of motor function on the exposed cerebral structures. ${ }^{16}$ Although it would be possible to perform both methods during one operation on the same patient, this would increase the duration of surgery. We perform intraoperative diagnostics using direct cortical stimulation as described, with repetitions of stimulation at different intensities in less that than 20 minutes, in order to limit operation time. The information gained by direct cortical stimulation is sufficient and can be obtained without the use of staff trained in electrophysiology.

Nowadays there is an abundance of literature about functional MRI (fMRI) results and its applications, but only little research has been done comparing fMRI with intraoperative stimulation results. Differences between these methods should, however, be kept in mind. Intraoperative cortical electric stimulation leads to a direct motor phenomenon via neuronal activation. ${ }^{17}$ Functional MRI deals with a flow phenomenon secondary to neuronal activation. In the iterature, the spatial resolution of fMRI ranges up to $20 \mathrm{~mm} .^{18}$ Although a good correlation between the stimulated motor hand area and fMRI activity has been found, ${ }^{19}$ fMRI has its limitations in paradigm selection, and time intensity of examination and data preperation. Although, in our opinion, fMRI seems to be a very good, non-invasive screening method, it is very difficult or impossible to selectively activate all functional areas surrounding a small subcortical lesion $<30 \mathrm{~mm}$ in size and the fibre tracts surrounding this lesion. Therefore, we think that implementation of fMRI data in the surgical microscope does not provide as much information as functional cortical mapping. Similarly, in a previous PET study on 
cerebral language activation areas, only $65 \%$ correlation was found between PET data and intraoperative language monitoring in awake surgery. ${ }^{20}$

The different functional stimulation results between cortical stimulation and stimulation of the resection cavity in three patients can be explained by the fact that the descending pyramidal fibre tracts converge, bringing neighbouring functions closer together. Reaching a subcortical lesion sometimes requires careful dissection to separate these fibres without damage, an achievable goal as only one of the 10 patients had a surgery induced motor disturbance. Because Penfield's homunculus shows the foot elevating function medially to the hip bending region, this patient's problem probably occurred as a result of coagulation on the medial resection cavity wall during the final haemostasis.

In all patients the Surgiscope was very useful in determining the site of the craniotomy and in localising the tumour. An additional advantage compared with other neuronavigation systems is the fact that the robotic microscope is able to "remember" certain positions or anatomical structures, similarly to a stereotactic targeting device. ${ }^{11}{ }^{21}$ Obviously, when using the Surgiscope, as with all stereotactic procedures in the brain, brain shift cannot be ignored. By the minimally invasive approach described, however, brain shift did not have a significant effect on tumour localisation.

\section{Authors' affiliations}

W Eisner, J Burtscher, K Twerdy, Department of Neurosurgery, University of Innsbruck, Anichstrasse 35, A-6020 Innsbruck; Austria

R Bale, Department of Radiology I, University of Innsbruck

F Koppelstätter, S Golaszewski, Department of Radiology II, University of Innsbruck

R Sweeney, Department of Radiotherapy-Oncology, University of

Innsbruck

C Kolbitsch, Department of Anaesthesia and Intensive Care, University of Innsbruck

\section{REFERENCES}

1 Apuzzo MLJ. Brain surgery. Complication avoidance and management. Vol 1. Churchill Livingstone, New York. 1993:379-90.

2 Ebeling U,Schmid DU, Reulen HJ. Tumor surgery within the central motor strip: surgical results with aid of electrical cortical motor cortex stimulation. Acta Neurochir (Wien) 1990;101:110-17.
3 Eisner W, Reulen HJ, Ilmberger J, et al. Intraoperative mapping of eloquent brain areas. Frontiers of radiation. Therapy and oncology. Zürich: Karger Verlag, 1999;33:28-36.

4 Haase J. Neurosurgical tools and techniques: modern image-guided surgery. Neurol Med Chir 1998;38(suppl):303-7.

5 Steude U, Hamburger C. Preoperative stereotactic localisation of cerebral tumortumours: a new tool to improve microsurgical tumortumour removal. App/ Neurophysiol 1987;501:241-2.

6 Reulen HJ, Schmid DU, Ilmberger J, et al. Intraoperative identification of motor and speech functions: The munich protocol. In: Siegenthaler W, Haas R, eds. In the decade of the brain. Stuttgart: Georg Thieme Verlag 1995:58-60

7 Oldfield RC. The assesment and analysis of handedness: the Edinburgh inventory. Neuropsychologia 1971;7:97-113.

8 Eljamel MS. Frameless stereotactic neurosurgery: two steps towards the holy grail of surgical navigation. Stereotact Funct Neurosurg 1999;722:125-8.

9 Apuzzo ML, Chen JC. Stereotaxy, navigation and temporal concentration. Sterteotact Funct Neurosurg 1999;722:82-8

10 Muacevic A, Uhl E, März U, et al. When the computer is assisting the surgeon. Navigation system will also find small brain metastases. MMW Fortschr Med 2000;1426:40-2.

11 Haase J. Image-guided neurosurgery/neuronavigation/the surgiscope reflexions on a theme. Minim Invasive Neurosurg 1999:42;53-9

12 Penfield WP, Boldrey E. Somatic motor and sensory representation in the cerebral cortex of man as studied by electrical stimulation. Brain 1937:60:389-443.

13 Ebeling U, Reulen HJ. Space-occupying lesions in the sensori-motor region. Adv Tech Stand Neurosurg 1995;22:137-81.

14 Ojemann GA, Ojemann J, Lettich E, et al. Cortical language localization in left, dominant hemisphere. An electrical stimulation mapping investigation in 117 patients. J Neurosurg 1989;71:316-26.

15 Wood CC, Spencer DD, Allison D, et al. Localization of human sensorimotor cortex during surgery by cortical surface recording of somatosensory evoked potentials. J Neurosurg 1988:68:99-111.

16 Allison T, McArthy G, Wood CC, et al. Human cortical potentials evoked by stimulation of the median nerve. I. Cytoarchitectonic areas generating short latency activity. J Neurosurg 1989;62:694-710.

17 Zimmermann $M$. Electrical stimulation of the human brain. Human Neurobiology 1982;1:227-9.

18 Yetkin ZF, Mueller WM, Morris GL, et al. Functional MR activation correlated with intraoperative cortical mapping. AJNR Am J Neuroradiol 1997; 18:1311-15.

19 Yousry T, Schmid UD, Schmid D, et al. Topography of the cortical motor hand area: prospective study with functional MR imaging and direct motor mapping at surgery. Radiology 1995;195:23-9.

20 Herholz K, Reulen HJ, von Stockhausen HM, et al. Preoperative activation and intraoperative stimulation of language related areas in patients with glioma. Neurosurgery 1997;41:1253-62.

21 Eisner W, Steude U, Burtscher J, et al. Surgery of lesions in the motor strip combining a stereotactically guided mini-craniotomy with electrophysiological mapping of the motorcortex. Minim Invasive Neurosurgery 2001;44:230-4. 